\title{
Belphégor
}

\section{Weinstock, Jeffrey Andrew. The Monster Theory Reader. Capturer l'image changeante d'une altérité en perpétuelle reconfiguration}

\section{Théodore Dehgan}

\section{(2) OpenEdition}

\section{Journals}

Édition électronique

URL : https://journals.openedition.org/belphegor/4280

DOI : $10.4000 /$ belphegor.4280

ISSN : 1499-7185

Éditeur

LPCM

Référence électronique

Théodore Dehgan, « Weinstock, Jeffrey Andrew. The Monster Theory Reader. Capturer l'image changeante d'une altérité en perpétuelle reconfiguration », Belphégor [En ligne], 19-2 | 2021, mis en ligne le 07 janvier 2022, consulté le 20 septembre 2022. URL : http://journals.openedition.org/ belphegor/4280 ; DOI : https://doi.org/10.4000/belphegor.4280

Ce document a été généré automatiquement le 20 septembre 2022.

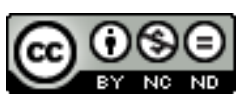

Creative Commons - Attribution - Pas d'Utilisation Commerciale - Pas de Modification 4.0 International - CC BY-NC-ND 4.0

https://creativecommons.org/licenses/by-nc-nd/4.0/ 


\title{
Weinstock, Jeffrey Andrew. The Monster Theory Reader. Capturer l'image changeante d'une altérité en perpétuelle reconfiguration
}

\author{
Théodore Dehgan
}

\section{RÉFÉRENCE}

Weinstock, Jeffrey Andrew (dir.). The Monster Theory Reader. Minneapolis : University of Minnesota Press, 2020.

L'ouvrage The Monster Theory Reader, supervisé par Jeffrey Andrew Weinstock, déploie les enjeux et thématiques qui traversent la notion de monstruosité à travers vingtquatre articles compilés dans quatre parties thématiques. La "théorie du monstre", établie sous ce nom par Jeffrey Jerome Cohen, est définie par Weinstock comme un champ d'investigation auquel peuvent se rallier des chercheurs de domaines variés.

Ces différents domaines sont mis en lien thématiquement dans l'ouvrage. La première partie offre une boîte à outils théorique pour les études de monstres (Monster Theory Toolbox), en proposant des textes de Sigmund Freud, Masashiro Mori et Julia Kristeva. Et l'ouvrage se clôt sur une quatrième partie, The Promises of the Monster (Les Promesses du Monstre), présentant une série de recadrages épistémologiques qui nous incitent à embrasser la figure du monstre comme une échappatoire aux normes établies (avec des textes d'Anthony Lioi, Donna Haraway et Patricia MacCormack). Ces articles qui ouvrent et ferment le recueil se distinguent des autres par leur volonté de faire du concept du monstre un absolu susceptible d'être déraciné. Les articles répartis dans les sections Monsterizing Difference (rendre la différence monstrueuse) et Monsters and Cultures (Monstres et Cultures) inscrivent quant à eux à chaque fois leurs monstres dans 
des champs analytiques spécifiques. Or, cette diversité des champs disciplinaires est décrite en introduction comme une difficulté :

Comme le monstre qu'elle théorise, la théorie du monstre transgresse les limites catégoriques, se répandant dans différentes disciplines. Ce que sont les monstres, d'où ils viennent, ce qu'ils signifient et les phénomènes culturels qu'ils engendrent, sont des questions qui ont préoccupé les philosophes, les théologiens, les psychologues, les physiciens et les critiques culturels. Parce que toutes les cultures ont leurs propres monstres, la théorie du monstre est nécessairement une entreprise internationale - laquelle, en gardant en tête les normes et les attentes culturelles changeantes, doit avancer avec prudence quand il s'agit de généraliser (le même monstre résonne différemment suivant les périodes et les régions) ${ }^{1}$.

3 Cette volonté de saisir le monstre à différentes époques et dans différentes aires culturelles en englobant des perspectives diverses échoue cependant en partie dans la mesure où, à l'exception d'un article sur le sens du Tanuki dans le Japon de la réouverture (Michael Dylan Forster) tous les textes rassemblés dans l'ouvrage se réfèrent à un contexte occidental (au moins partiellement comme avec Beasts from the Deep d'Erin Suzuki). Mais malgré cette limite de cadrage, le prisme du monstre permet d'introduire une forme de distance critique. Analyser le monstre qui remet en question les catégories cognitives et les stratégies d'interprétation établies ${ }^{2}$ revient systématiquement à dépasser des présupposés contextuels, occidentaux en l'occurrence.

4 Si les monstres sont les contrepoids qui définissent une normalité située dans le temps et l'espace, l'analyse des monstres apparait alors comme un outil de remise en question des catégories. Le monstre est défini par son intolérable ambiguïté (Elizabeth Grosz) et menace la compréhension du monde, de soi et de la relation entre les deux ${ }^{4}$, il opère en ce sens un recadrage sémantique qui permet de révéler des perspectives écartées par les a priori culturels et sociaux.

\section{Concepts du monstrueux}

5 La boîte à outils du Monster Theory Reader contient les articles les plus anciens et les moins spécifiques. L'influence de ces textes fondateurs se répercute dans le reste de l'ouvrage. On trouve dans cette partie le fameux texte de Freud sur l'inquiétante étrangeté, et celui de Masashiro Mori sur la «vallée de l'étrange». Le roboticien Masashiro Mori décrit la "Uncanny valley ", comme une théorie de design robotique selon laquelle plus un robot ressemblerait à un humain et plus ses défauts seraient monstrueux, jusqu'à être enfin assez ressemblant pour être accepté. Enfin, le concept du monstrueux développé par Julia Kristeva dans Approaching Abjection [Pouvoirs de l'horreur] est "l'abjection ». La notion se réfère à la réaction physique de dégoût et d'horreur ressentie suite à la perte de distinction entre sujet et objet ou entre soi et l'autre. Cette perte de distinction peut être aussi bien provoquée par la vue de cadavres que par la peau sur du lait chaud. L'abjection serait un rappel de violences liées à des logiques de séparation qui ont permis à l'humain de se définir comme sujet face à une altérité objectale. En utilisant l'abjection, des sociétés primitives ont déterminé le périmètre de leur culture en se distinguant des animaux. Mais l'abjection serait aussi un mouvement individuel universel, correspondant au moment où l'humain se sépare de sa mère et reconnaît une limite entre soi et l'autre. L'abjection menace les limites, notamment celles qui séparent le sujet de l'objet (la vue d'un cadavre nous rappelle 
notre matérialité). Selon Kristeva qui étend cette théorie à ses analyses d'œuvres (Louis-Ferdinand Céline en particulier), l'art seul purifie l'abjection par la catharsis.

6 L'inquiétante étrangeté, la vallée de l'étrange et l'abjection se rejoignent dans l'idée d'un monstre terrifiant car il bouleverse les limites de l'humanité. Ces trois articles en ce sens tentent de circonscrire l'indéfinissable. En fin d'ouvrage, dans la partie The Promises of Monsters (Les Promesses des Monstres), Anthony Lioi, Donna Haraway et Patricia MacCormack proposent également une conception du monstrueux qui nous permettrait d'échapper à des civilisations mortifères en redéfinissant notre système de perceptions. Dans of Swamp Dragons, Anthony Lioi appelle les environnementalistes à s'extirper de conceptions faisant de la nature un parc national immuable et grandiose, et de s'appliquer, en suivant des principes de justice environnementale, à s'occuper de la saleté ("dirt") : On devrait rendre à la saleté ce qui lui est dû̀. Il met en avant l'idée d'un Swamp Dragon, dragon des marécages, comme emblème de cet environnementalisme qui ne rejette pas l'impureté mais l'inclut et même l'embrasse. S'occuper des espaces diminués (diminished spaces) ou pollués permettrait ainsi de garder un œil sur le monstrueux, et d'anticiper ainsi sur de futurs dangers. Devenir le dragon du marécage équivaudrait à voyager à travers les cauchemars de la philosophie et les ruines de l'industrie et revenir en sachant aimer davantage le monde dans lequel on vit ${ }^{6}$.

7 Donna Haraway dans The Promises of Monsters, qui donne son titre à la dernière partie de l'ouvrage, questionne également le rapport de possession et d'exploitation entre l'humain et la nature à travers l'idée que nous ne naissons pas mais que " nous sommes faits $»^{7}$ par plusieurs acteurs dans ce qu'elle nomme un "artéfactualisme». Cet artéfactualisme permettrait de refuser le colonialisme, comme reproduction du même, et la fiction des lumières et de l'histoire des sciences et technologies comme paradigmes du rationalisme. Haraway écarte également l'hyperproductvité, qui refuse le pouvoir d'action à tous sauf un, mais aussi l'idée d'un pouvoir transcendant de la nature. Pour Haraway, la nature ne peut être dénaturée dans le sens où elle ne possède pas de premier état "pur ». Avec son carré d'artéfactualisme, Haraway présente une politique régénérative pour les inappropriés et impropres (les "autres", others). Ce carré est constitué de quatre espaces : réel (la terre), externe (extraterrestre), virtuel (la science-fiction), interne (le corps biologique). Haraway analyse pour chaque espace une image qui trahit une logique de domination, en révélant les acteurs secrets, les "monstres", effacés par les acteurs de l'hégémonie impérialiste patriarcale.

Dans la même veine que Donna Haraway, Patricia MacCormack développe l'idée d'une tératologie post humaine (Posthuman Teratology). Pour elle, le monstre, dans la mesure où il ne correspond pas à une catégorie mais où il se définit par ce qu'il n'est pas (en l'occurrence, humain), se rapproche du concept du post humain. Le monstre est défini par le contexte et jamais par lui-même. D'après MacCormack, selon les principes du posthumanisme, tous les humains sont des monstres car aucun ne correspond parfaitement à la définition de l'humanité, laquelle n'est qu'une idée. Le monstre posthumain de MacCormack refuse le temps narratif arbitraire qui est l'expression d'un pouvoir, l'humanisme pour lequel l'altérité est une faute, ou l'idéologie majoritaire qui tend à réitérer un certain mode de pensée au lieu de penser par soi-même. Son concept de monstre posthumain se définit aussi en négatif en refusant à la fois la fétichisation de l'animalité et l'éternité transhumaine. La tératologie posthumaine consiste ainsi à se revendiquer comme monstre soi-même afin de se passer d'une altérité repoussoir pour se définir en tant qu'humain constitué d'éléments d'altérité. 


\section{Typologies du monstrueux}

La monstruosité est une catégorie épistémologique lâche et flexible qui laisse une place aux définitions de ce qui complique ou semble résister à la définition ${ }^{8}$.

9 Le monstre est donc largement défini conceptuellement comme une menace qui perturbe les limites et les catégories. Pourtant, force est de constater qu'une part non négligeable du Monster Theory Reader entend faire la typologie des monstres suivant différentes catégorisations. La première de ces typologies est celle des études de monstres. Dans son introduction, Jeffrey Andrew Woodstock définit différents champs d'étude consacrés au monstre. Tout d'abord, la tératologie avec ses sous-catégories de "freaks" et ses théories plus ou moins surnaturelles, la mythologie subdivisée chronologiquement en "races monstrueuses", "créatures mythiques" et "cryptides", et enfin, dernière catégorie du monstrueux, la psychologie qui déplace la monstruosité de l'apparence vers l'intériorité.

10 Cette première catégorisation ouvre déjà des perspectives plus contextuelles. Notamment la sous-catégorie de la tératologie et ses « freaks » qui est développée dans son article par Elizabeth Grosz.

11 L'article qui donne son nom au Monster Theory Reader, Monster Culture (Seven Theses) de Jeffrey Jerome Cohen, n'est pas à proprement parler une catégorisation de monstres mais apparaît davantage comme une dissection épistémologique du concept du monstre. La première thèse avance que «le corps du monstre est un corps culturel » (the monster body is a cultural body) et que le monstre signifie toujours quelque chose d'autre que lui-même en incarnant un certain moment culturel. La deuxième thèse : "le monstre d'échappe toujours" (The monster always escapes) développe l'idée que le sens du monstre est volatile : Dracula n'a pas le même sens au XIXème et XIXème siècle. Il revient toujours dans des habits différents. Dans «Le monstre est un signe avantcoureur de la crise des catégories" (The monster is harbinger of category crisis) Cohen explique que la présence du monstre entraîne une crise et un questionnement des catégories connues.

Dans la même lignée, «le monstre demeure au seuil des différences " (The monster dwells at the gate of differences) établit que des différences politiques, idéologiques, de genre ou de race, sont des catalyseurs parfaits pour créer un monstre et justifier la colonisation et l'oppression. Ces monstres sont des " démons nourris à la propagande " (propaganda bred demons) et des boucs émissaires. "Le monstre police les frontières du possible» (The monster polices the border of the possible) distingue les monstres politiques invitant ou justifiant une action violente et les monstres de prohibition qui gardent les frontières en jouant le rôle de menaces ou de fables édificatrices. Le monstre, parce qu'il pose des limites, maintient en place les valeurs de la société. Le monstre est le refoulé qui doit être exilé.

«La peur du monstre est en réalité une sorte de désir » (The fear of the monster is really a kind of desire), la sixième thèse commence par établir l'idée que le monstre est lié à des pratiques interdites pour normaliser ou renforcer les règles mais qu'il attire aussi pour cette raison. Il est une échappatoire à la contrainte. Il est méprisé mais envié pour sa liberté et se situe dans un espace ambigu entre attirance et dégoût. La dernière thèse : « le monstre est toujours sur le point de devenir » (The monster stands at the threshold of becoming), conclut l'essai en répondant à la question de savoir si les monstres existent : 
ils existent, sinon comment pourrions-nous exister ?' Pour Cohen, les monstres questionnent nos présupposés. Nous les repoussons mais ils reviennent demander pourquoi nous les avons créés. C'est à cette question que l'ensemble du Monster Theory Reader tente de répondre.

Les deux derniers articles de la boîte à outils du Monster Theory Reader offrent d'autres propositions de classification du rôle joué par les monstres. Dans An Introduction to the American Horror Film, Robin Wood énumère les différentes formes d'altérité monstrueuse représentées par les monstres du cinéma américain (la sexualité des femmes, le prolétariat, d'autres cultures, d'autres groupes ethniques dans la culture, des idéologies politiques alternatives, une déviation des normes sexuelles, enfin les enfants, être insaisissables et horrifiques ${ }^{10}$ ). En ce sens, selon Robin Wood, une fin heureuse dans un film d'horreur est souvent un retour à la répression. Mais les monstres des films d'horreur américains peuvent aussi porter une critique de la norme, spécifiquement après les années soixante et les mouvements des droits civiques. D'après Wood, depuis Psychose (Alfred Hitchcock, 1960) l'horreur peut être américaine et familiale. Définir ce que représente le monstre dans un film d'horreur permet selon Wood de cerner si le film est progressiste ou réactionnaire mais la limite établie entre les deux n'est jamais claire. Néanmoins, Wood donne quatre éléments permettant d'identifier à priori un film d'horreur réactionnaire. Tout d'abord, quand le monstre est désigné comme absolument néfaste sans aucune ambiguïté, le film a de plus grandes chances d'être réactionnaire. Les monstres sont destructeurs par définition mais quand cette destruction est justifiée ou expliquée dans le récit, le film est le plus souvent progressiste. La présence du christianisme dans les films, quand il est présenté comme une force positive, est aussi un indice pour identifier un film d'horreur réactionnaire. Les films présentant les monstres comme totalement inhumains et ne suscitant pas la moindre sympathie sont également plus réactionnaires (Wood évoque les films anticommunistes des années du Maccarthysme). Enfin, pour Wood, la confusion entre la sexualité réprimée et la sexualité elle-même (les deux étant alors présentés indistinctement comme monstrueuses) est symptomatique du film d'horreur réactionnaire qui témoigne d'une société où la peur du sexe est implantée dès l'enfance.

Dans Fantastic Biologies and the Structures of Horrific Imagery, Noël Carroll a pour ambition de nous livrer un guide pour créer une image de monstre. Il définit le monstre comme impur puisqu'il contredit les catégories connues. Mais il saisit ce qui fait le monstrueux en le définissant selon cinq conditions : la fusion de deux qualités en théorie irréconciliables, par exemple un mort qui est aussi vivant, un agglomérat de parties disparates, comme la créature de Frankenstein, la fission (une division dans le temps qu'on retrouve par exemple chez les loups-garous et les métamorphes), la magnification (une créature dont on amplifie la taille devient un monstre), la massification (un essaim d'êtres grouillants devient vite effrayant) et enfin la métonymie horrifique (en entourant le monstre d'éléments qui créent des phobies, on amplifie son aura d'horreur : Norman Bates a l'air d'un homme ordinaire mais il est entouré d'objets inquiétants qui annoncent sa monstruosité dans Psychose).

16 Enfin, dans la troisième partie de l'ouvrage, Monsters and Culture, Jeffrey Andrew Weinstock recatégorise les monstres dans une époque spécifiquement contemporaine avec son article Invisible Monsters, Vision, Horror, and Contemporary Culture. En partant du postulat que les monstres sont liés à la culture qui les crée, il détaille quatre types de 
monstres contemporains qui sont tous invisibles : le psychopathe (ou le terroriste) qui pourrait être n'importe qui, les corporations ou agences gouvernementales sans visage (tout le monde est un monstre), le virus qui s'infiltre, et la nature anthropomorphique qui prendrait sa revanche sur l'homme (le monstre est partout).

\section{Les monstres en contexte}

17 Andrew Weinstock ouvre son article Invisible Monsters sur l'idée qu'il faut un village pour faire un monstre; et ce village, contexte métaphorique qui crée des monstres sociétaux, est analysé dans les articles qui constituent la majorité de l'ouvrage. Les monstres du Monster Theory Reader émergent de lieux et d'époques diverses.

En tant que figures repoussoirs correspondant à ce qui ne définit pas un être humain normé, les monstres naissent de la xénophobie (monstres politiques justifiant des guerres), plus précisément de l'antisémitisme (Gog et Magog), du racisme (les monstres reflets du colonialisme), de l'islamophobie (le terroriste), mais aussi du validisme (les "freaks »), du sexisme (féminin monstrueux) et de l'homophobie (les monstres queers). Ils reflètent aussi des questions environnementales (peur du nucléaire, nature vengeresse) et enfin éthiques (en lien avec des questions de psychanalyse, de psychologie ou de religion). Et ces mythes monstrueux ont un impact sur la réalité des groupes ciblés, entérinant et justifiant des abus et des inégalités existantes.

Ainsi, dans son article Monstrous Strangers at the Edge of the World: The Monstrous Races, Alexa Wright analyse les "races monstrueuses" de la Bible d'Arnstein de Lunandous d'Arnstein. Ces monstres humains manifestent pour Wright les interrogations culturelles de l'époque sur les limites entre nature et culture, entre l'humain et le non humain. Ces races monstrueuses, souvent hybrides (hommes à tête de chien) excitent la curiosité des lecteurs tout en se voyant affecter une connotation négative. Elles remontent à des textes de l'antiquité grecque et romaine et ont été transmises au Moyen-Âge. A cette époque, l'aspect monstrueux de ces races était expliqué par des vices afin de rationaliser le monstre en entérinant et essentialisant des lois culturelles. De même, il était établi qu'avoir un corps humain ou non était une condition pour avoir une âme humaine. Pour Wright, les races monstrueuses ont cet intérêt qu'elles montrent en occident la tendance historique à se définir face à une altérité au corps et comportement étranges.

Jack Halberstam revient quant à lui sur le type de monstre ayant émergé de la littérature gothique du XIXe siècle dans son article Parasites and Perverts: An Introduction to Gothic Monstrosity. Selon Jack Halberstam, les monstres de la littérature étaient associés à des connotations antisémites et racistes mais aussi sexuelles, les trois pouvant s'articuler. Il cite les analyses d'Eve Kosofsky Sedgwick dans Gothic Conventions an Aesthetic of Pleasurable Fear. Pour Halberstam, il existe un rapport ambigu dans les histoires gothiques entre le plaisir et la censure portée par le récit qui condamne clairement les agissements monstrueux. A l'époque où a émergé le roman gothique, avec le déclin de l'aristocratie, il existait en Angleterre un besoin spécifique d'établir des critères de communauté et de supériorité. Le clergé et l'aristocratie sont, dans les récits gothiques, corrompus par le monstrueux alors que le monde bourgeois et son modèle de sexualité de reproduction est mis en avant. Le sang de la noblesse est devenu le sang du natif anglais opposé à celui des races impures. Pour Jack Halberstam, Dracula, qui est caractérisé comme un étranger sexualisé, est une menace pour la 
femme anglaise. La sexualité parasite et non reproductive a un caractère non anglais. Le monstrueux réprimé que décrit Freud devient, dans ce contexte, étranger. Pour Sedgwick, la menace est l'homosexualité gothique mais pour Halberstam, il ne s'agit que d'une partie du problème. En réalité, le monstre est un paria racial. Les monstres représentent ce que l'humain n'est pas, et l'humain dans l'Angleterre victorienne était devenu un homme blanc hétérosexuel de la moyenne bourgeoisie à la sexualité reproductive.

21 Jon Stratton avance dans Zombie Trouble: Zombie Texts, Bare Life, and Displaced People que les zombies qui se sont multipliés dans les médias des années 2000 reflètent la réalité des peuples déplacés : des immigrants dans l'illégalité, des réfugiés et des demandeurs d'asile. Dès 1915, les zombies sont une métaphore des aliénés d'après Stratton qui avance qu'ils étaient des travailleurs esclaves dans une économie capitaliste. Il cite Colin Dayan selon laquelle les zombies racontent l'histoire de la colonisation à travers la métaphore d'humains exploités réduits à l'état d'objets. Les zombies représentent alors la destruction de la culture haïtienne par le colonialisme américain déguisé en développement. Depuis Romero, le zombie n'est plus au service d'une personne précise qui l'a créé, ce qui était le cas à l'origine dans le mythe populaire des sorciers vaudous. Avec Night of the Living Dead (George A. Romero, 1968), Romero a mis en place l'idée d'une apocalypse zombie. Ces apocalypses sont très présentes dans la pop culture des années 2000 , dans laquelle les créatures représentent souvent la menace d'une révolte des opprimés contre la société occidentale. Dans certains exemples analysés par Stratton, Orgueil, Préjugés et Zombies (Seth Grahame-Smith, 2009), l'apocalypse zombie représenterait même plus spécifiquement une révolte d'esclaves.

Saisissant un corpus incluant des médias du début du XXème siècle au milieu des années 2000, Annalee Newitz analyse la blanchité dans les histoires d'horreur liées à la race dans The Undead: A Haunted Whiteness. Au début du XXème siècle, dans les récits de morts-vivants on devine en filigrane le motif du colonialisme et de l'esclavage où les blancs, hantés par un passé où les personnes racisées étaient libres et puissantes, anticipent un futur où ils seront eux-mêmes les fantômes de leur grandeur déchue alors que les anciens colonisés retrouveront leur souveraineté. H. P. Lovecraft, en faisant des racisés des aliens, "les dieux anciens" qui rendent les humains fous, préfigure cette chute d'un colonialisme hanté par le passé. Selon Newitz, l'immortalité des dieux anciens est à assimiler aux traditions des minorités qui continuent de subsister aux États Unis. Newitz cite aussi le film Birth of a Nation où les membres du Ku Klux Klan ressemblent à des fantômes d'un passé colonial déjà révolu.

Dans un contexte postcolonial, c'est le passé colonial blanc qui hante le présent et figure l'ennemi. Mais d'autres tensions émergent entre les héros noirs de classes moyennes et pauvres, et sont visibles dans les films de zombies et de blaxploitation qu'analyse Newitz ; Blacula (William Crain, 1972), Nightbreed (Clive Barker, 1990), The People under the stairs (Wes Craven, 1991) et Tales from the Hood (Rusty Cundief, 1995), un film où le désir de pouvoir au détriment de la communauté conduit des personnes noires de classe moyenne aux enfers où se trouvent les propriétaires blancs d'esclaves. Enfin, Newitz pointe les questions de métissage dans les films d'horreur Blade et Underworld et les tensions qui en résultent.

Dans un contexte d'Amérique post 11 septembre 2001, Jasbir K. Puar and Amit S. Rai reviennent sur la figure du "pédé terroriste" (terrorist fag) dans Monster, Terrorist, Fag: The War on Terrorism and the Production of Docile Patriots. Ils rapprochent le terroriste 
moderne des monstres raciaux et sexuels des XVIIIème et XIXème siècles. La construction du terroriste est basée sur l'idée de perversité sexuelle qui représente un échec à l'hétérosexualité et donc une monstruosité queer. Par opposition est construite une hétérosexualité patriotique dominante dans les médias américains. Après les attentats du 11 septembre, des représentations de Ben Laden violé par l'Empire State Building sont apparues très rapidement et cette image du terroriste "oriental" dépravé violé par l'Amérique hétérosexuelle perdure. Cette imagerie émascule l'ennemi et le rend "autre" en le queerisant. Outre cette fabrication d'une altérité héritée d'une imagerie coloniale orientaliste, la méconnaissance du contexte d'où émerge le terrorisme islamiste amène à de nombreux contre-sens d'après Puar et Rai. Les études du terrorisme aux Etats Unis se basent sur des clichés de psychanalyse occidentale et réduisent les enjeux complexes, liés aux racines historiques du terrorisme, à de la frustration sexuelle et à une mauvaise mère. Ils excluent systématiquement les questions d'économie politique et classent les terroristes selon des notions familières à l'occident.

Outre le colonialisme, le racisme et l'islamophobie, l'antisémitisme a modelé de nombreux monstres dans la culture occidentale. Dans Blood, Jews, and Monsters in Medieval Culture, Bettina Bildhauer analyse des textes religieux du XIIIème siècle qui ont contribué à créer un monstre juif : la carte d'Ebstorf (Mappa Mundi) et deux sermons du franciscain Berthold de Regensburg. Elle cite les Gog et Magog visibles sur la carte d'Ebstorf et décrits comme des géants cannibales qui accompagneront l'antéchrist et qui sont pour le moment cachés derrière un mur bâti par Alexandre le Grand. Les Gog et Magog sont des prostitués et des démons mais surtout des juifs. Berthold de Regensburg quant à lui compare les juifs à des monstres de l'Apocalypse dans ses sermons. Mais ces monstres font partie du plan de Dieu. En ce sens, selon Bildhauer, ce qui crée la peur n'est pas la judéité en soi mais le fait que les juifs et les chrétiens ne sont pas des catégories si distinctes et qu'il y a de l'hybridité dans les deux. Les juifs rappellent les passions du christ tout comme les Gog et Magog montrent la puissance de Dieu. Ils sont en ce sens indissociables.

La question du corps «monstrueux » est abordée frontalement par Elizabeth Grosz et Margrit Shildrick dans leurs articles respectifs : Intolerable Ambiguity: Freak as/at the Limit et The Self's Clean and Proper Body. Les deux articles analysent le traitement médiatique et médical de "freaks " spécifiquement des cas des jumeaux fusionnés.

Elizabeth Grosz commence dans son article par faire une typologie des freaks montrés dans des cirques aux XIXème et XXème siècles. Elle les définit comme des êtres de fusion ; les hommes animaux, les siamois, les enfants sauvages (fusion de la nature et de la culture), les femmes à barbe et les personnes dites hermaphrodites intersexes ou maquillées (fusion des deux sexes), les «nains» (personnes de petite taille présentées comme des adultes/enfants), les géants (fusion des humains et des dieux) et les humains squelettiques (à la fois morts et vivants). Dans son article, Grosz s'attarde plus spécifiquement sur les questionnements que soulèvent les personnes « hermaphrodites" (intersexes) et les jumeaux "siamois " (fusionnés). Ces personnes excitent la curiosité sexuelle et brouillent les limites entre les sexes et entre les êtres. Grosz observe que cette curiosité et ce trouble qui amènent à une insécurité dans l'identité suscitent une volonté médicale de faire rentrer ces corps qui brouillent les limites dans la norme alors que les personnes intersexes et les jumeaux fusionnés ne le désirent pas et s'attristent même à cette idée. 

concentre sur le cas des jumeaux fusionnés qui remettent en question les définitions philosophiques de ce qui constitue un humain. Elle met en avant que le corps normé sans âge et universel n'existe pas et que les différences de morphologies mettent toujours à mal la norme. L'existence de la monstruosité, d'après elle, sert à définir par comparaison la corporalité délimitée et sécurisée de la majorité. Mais le corps «standard" n'est pas en soi normal mais normatif. Les idéaux du corps parfait à l'autonomie inviolable amènent ainsi à la nécessité « éthique » de séparer des jumeaux fusionnés dont elle étudie un cas ayant conduit à la mort d'une des deux jumelles qui était pourtant en bonne santé avant l'opération. Les articles de Grosz et Shildrick questionnent l'arbitraire de ces retours forcés à la norme pour préserver les catégories cognitives connues dans un statu quo confortable.

Monstrous-Feminine: An Imaginary Abjection, Barbara Creed analyse une horreur symboliquement et corporellement féminine opposée à une norme de corps masculine. En remontant à Freud et à Kristeva (peur de la castration et abjection de la séparation avec la mère), elle analyse l'aspect phallique des sorcières et de la Méduse qui fige les hommes d'un regard, les rendant "durs»; elle se penche également sur des films opposant une figure maternelle monstrueuse et sa fille qui doit la rejeter comme dans Carrie au bal du diable (Brian De Palma, 1977) et Alien (James Cameron, 1979).

30 Ces analyses de corps monstrueux aboutissent à des questions de sexualité frontalement abordées par Harry Benshoff dans The Monster and the Homosexual où il est question des monstres queers émergeant de sociétés occidentales hétéronormées. Avant d'analyser Hollywood, Benshoff revient sur le modèle du roman gothique où l'homosexualité est le propre d'une aristocratie qui cherche le plaisir par tous les moyens. Les hommes efféminés mis en scène dans les textes d'Horace Walpole, M.G. Lewis, Oscar Wilde, Sheridan Le Fanu, Mary Shelley, Lord Byron et Polidori, seraient appelés queers selon les standards actuels. Ils représentent une menace constituée par l'homosexuel présenté comme décadent, élitiste et lié à la mort. Pour Benshoff, la romance hétéropatriarcale est interrompue à Hollywood par le personnage queer dans les films d'horreur. Si le film hollywoodien fait du queer un monstre, il invite aussi à pénétrer la subjectivité du monstre en filmant de son point de vue, et il peut donner au monstre des motivations plus complexes qu'au couple hétérosexuel qu'il va tuer. En outre, dès la fin des années 1960, le cinéma hollywoodien offre des représentations de personnes queers qui sont aussi parfois des victimes. Comme les «scientifiques fous » qui veulent restructurer la société, les queers attaquent la notion dominante de nature mais aident aussi à construire une norme. Sans les gays, les hétéros ne sont pas hétéros ${ }^{11}$.

Si les monstres servent à l'établissement d'une norme blanche masculine hétérosexuelle, ils peuvent aussi questionner des comportements éthiques concrets. Pour Stephen T. Asma dans Monsters and the Moral Imagination, les récits de monstres fonctionnent comme des jeux de rôles moraux : «et si..." une apocalypse zombie survenait, comment se comporterait-on? Ils mettent à l'épreuve des convictions éthiques en contexte réel ou imaginaire. C'est ce qui explique le «victim blaming »fait de blâmer les victimes d'une agression - que Stephen T. Asma analyse à travers le cas d'une attaque au couteau dans un restaurant de New Haven, Connecticut, en 1994 et de son traitement médiatique. Cette situation d'irruption d'un "monstre " dans la normalité conduit à une projection dans un scénario imaginaire «et si ». L'idée de 
rester inactifs et vulnérables en cas d'attaque s'avère alors intolérable. Selon Asma, les monstres mettent en avant la vulnérabilité humaine; et tant que la vulnérabilité humaine existera, les monstres subsisteront ${ }^{12}$.

Dans Introduction to Religion and its Monsters, Timothy Beal entame une analyse du monstre sous un prisme chrétien. Le monstre est souvent lié à une horreur théologique, il est une réponse ou un avertissement, divin ou démoniaque. L'expérience de l'extase divine rencontre une telle équivocité, au sens où elle est décrite comme terrible et merveilleuse. C'est ce que Beal qualifie d'horreur théologique (theological horror ${ }^{13}$ ). Beal s'attarde d'abord sur Frankenstein dans lequel l'homme, égal de dieu, crée un monstre. Citant Nietzsche (qui combat les monstres peut en devenir un), il note que le lecteur tend davantage à s'identifier au monstre qu'à son créateur ou aux tueurs de monstres. Dans l'épigraphe de Frankenstein, Shelley cite Paradise Lost de John Milton (1667) dans lequel l'homme demande au créateur pourquoi il l'a créé. Au final, le monstre est davantage le créateur que la créature, et Timothy Beal en vient à se demander si être un dieu signifie être monstrueux.

Sortant en partie du contexte occidental, Erin Suzuki analyse dans Beasts from the Deep trois blockbusters américains de l'été des années 2010 en les mettant en parallèle avec les relations entre les Etats-Unis et l'Asie de l'Est. En effet, peu avant la sortie de ces blockbusters était annoncé aux Etats-Unis le projet de changer le centre de gravité de la politique étrangère américaine du Moyen-Orient à l'Asie du pacifique dans l'idée de contenir l'influence de la Chine et de garder la mainmise sur la politique globale. Dans les blockbusters estivaux qui font suite à cette annonce, les films de monstres des années de guerre froide où s'exprimait la peur du nucléaire sont revisités. Les monstres des films Battleship (Peter Berg, 2012), Pacific Rim (Guillermo Del Toro, 2013) et Godzilla (Max Borenstein, 2014), en plus d'être des allégories de la militarisation et de la libéralisation des nations qui dominent le pacifique, représentent les crises chaotiques et imprévisibles qui sont intrinsèquement liés à ces cadres néolibéraux qui ne peuvent pas les contenir. Les trois blockbusters cités présentent une victoire de technologies et d'alliances militaires transpacifiques (spécifiquement américano-japonaises) sur la menace des monstres aquatiques. Ces représentations restent néanmoins très ambivalentes, entre la glorification militaire et la conscience partielle des conséquences de cette militarisation sur les peuples autochtones invisibilisés et sur l'environnement. L'hybridation avec les aliens (par contact ou par fusion via le "drift " dans Pacific Rim) est aussi présentée dans ces récits comme nécessaire car elle permet d'acquérir une connaissance de l'ennemi qui mène à la victoire. Le drift, la fusion, serait l'ouverture radicale salvatrice à l'altérité alien et à son potentiel chaotique.

Quittant définitivement le contexte occidental, Michael Dylan Foster analyse les récits des Tanuki durant l'industrialisation du Japon et leur signification dans Haunting Modernity: Tanuki, Trains, and Transformation in Japan. Durant la restauration de l'ère Meiji débutée en 1868, qui a été synonyme d'une industrialisation du pays et de sa réouverture au commerce international, les récits de Tanuki ont été synonymes d'une confrontation entre le monde de la nature et des croyances populaires d'une part, et le désir de modernisation de la nation d'autre part. Les Tanuki, chiens de prairie polymorphes, sont par leur nature plastique adaptés à de nouveaux récits et ont donc évolué avec la modernité et la rationalisation scientifique. A travers un récit populaire où le Tanuki se change en train et finit écrasé par un autre train, se joue la transformation par la technologie des chemins de fer de paysages difficiles d'accès 
chargés de connotations mystiques. Le Tanuki hante néanmoins le pays transformé à travers son image populaire déclinée partout et ses capacités de métamorphe qui rappellent un passé pré-moderne. On retrouve d'ailleurs les Tanukis dans le film Pompoko (Isao Takahata, 1994) où ils mettent leurs pouvoirs de transformation en œuvre pour ramener pour un temps l'image du paysage préindustriel.

Dans ces deux derniers articles, Monsters from the Deep et Haunting Modernity, le monstre est déplacé dans d'autres temps et d'autres espaces et en changeant de contexte, il change de signification. Le Tanuki d'un Japon préindustriel n'a pas le même sens que le celui des XXe et XXIe siècles. De même, le Godzilla du Japon des années cinquante n'a plus du tout les mêmes connotations quand on l'importe dans les Etats Unis des années 2010. Le contexte d'où émerge la monstruosité est donc capital et la définition même de monstruosité doit être suffisamment plastique pour éviter les contre-sens.

Malgré la limite qu'implique la perspective occidentale et l'assemblage parfois disparate des articles et de leurs champs disciplinaires multiples, le Monster Theory Reader parvient à structurer un ensemble en cohérence avec son sujet. Ces acteurs divers peuvent, comme les monstres d'Haraway, constituer une unité critique de mise à distance des normes. Le recueil ouvre un large panel d'études de monstres en donnant la mesure du rôle joué dans nos imaginaires par ces expressions d'une altérité aussi effrayante que fascinante, comme une force ambiguë qui définit autant qu'elle remet en question les catégorisations sociétales.

\section{NOTES}

1. "Like the monster it theorizes, monster theory transgresses categorical boundaries, spreading out into different disciplines. What monsters are, where they come from, what they mean, and the cultural work they do are questions that have preoccupied philosophers, theologians, psychologists, physicians and cultural critics. Because all cultures have their own monsters, monster theory is by necessity an international endeavor-and one that, bearing in mind shifting cultural norms and expectations, must tread carefully when it comes to broad generalizations (the same monster resonates differently in different times and places) ». Jeffrey Weinstock, p. 1

2. «[...] challenges established cognitive categories and interpretive strategies ». p. 3

3. "Intolerable ambiguity "

4. "The monster is that which threatens understanding of the world, the self, and the relations between the two ». p. 3

5. «Dirt should be given its due ». Anthony Lioi, p. 439

6. «Travel(ed) into the nightmares of philosophy and the ruins of industry and return(ed) with a stronger way to love the world we actually live in ». Anthony Lioi, p. 452

7. «We are made ». Donna Haraway, p. 463

8. "Monstrosity is a loose and flexible epistemological category that allows us a space to define that which complicates or seems to resist definition ". Andrew Weinstock, p. 4

9. «Surely, they must, for if they did not, how could we? ». Jeffrey Jerome Cohen, p. 52

10. Robin Wood, p. 114 
11. "Without gays straights are not straight ». Simon Watney, Policing Desire: Pornography, AIDS and the Media (Minneapolis: University of Minnesota Press, 1987) cité par Harry Benshoff, p. 228

12. "[...] the monster concept is still extremely useful, and it's a permanent player in the moral imagination because human vulnerability is permanent ». Stephen T. Asma, p. 293

13. Timothy Beal, p. 296

INDEX

Mots-clés : horreur, monstres, Jeffrey Andrew Weinstock, Sigmund Freud, littérature d'horreur

AUTEUR

THÉODORE DEHGAN

Université Paris Nanterre 